\title{
Ecocardiografia Fetal. Apresentando o Método
}

\author{
Solange Bernardes Tatani
}

São Paulo, SP

O estudo do desenvolvimento do feto pela ultrasonografia, há vários anos vem sendo feito extensivamente, e tem sido de considerável ajuda na avaliação dos padrões de crescimento fetal e no diagnóstico de malformações congênitas. Entretanto, inicialmente, menor destaque foi dado à possibilidade de diagnosticar alterações na estrutura e função do sistema cardiovascular fetal.

Até há poucos anos, o único aspecto da fisiologia cardiovascular fetal, que podia ser rotineiramente monitorizado, era a freqüência cardíaca. Com a introdução da ecocardiografia (ECO) modo-M, bidimensional e as técnicas do Doppler (especialmente o mapeamento de fluxo a cores) no estudo do feto, foram permitidas a descrição acurada da anatomia intracardíaca, a análise seqüencial das câmaras e, finalmente, o reconhecimento de malformações, de distúrbios da função e do ritmo cardíacos no período pré-natal.

Os batimentos cardíacos fetais podem ser visibilizados pela ECO bidimensional, já na $6^{\mathrm{a}}$ semana de gestação, mas uma análise estrutural adequada geralmente só é factível a partir da $16^{\mathrm{a}}$ semana. Nesta idade gestacional, o coração fetal é ainda muito pequeno e, muitas vezes, não é possível um estudo completo ${ }^{1}$. A época ideal para a visibilização do coração fetal estende-se da $18^{\mathrm{a}}$ à $24^{\mathrm{a}}$ semana gestacional, quando o concepto está envolto por grande volume de líquido amniótico. Já no $3^{\circ}$ trimestre, a coluna vertebral fetal é freqüentemente anterior e as costelas são mais calcificadas, "sombreando" a área cardíaca e podendo dificultar a adequada avaliação do coração fetal nesta fase gestacional.

O exame cardíaco fetal consiste de um estudo sistemático e cuidadoso do situs atrial e das conexões veno-atrial, atrioventricular e ventrículo-arterial. Envolve, igualmente, avaliação adequada das paredes miocárdicas, septos atrial e ventricular, além dos arcos aórtico e ductal ${ }^{2}$.

O exame inicia-se pela determinação da posição do coração e do situs atrial. A posição do coração no tórax fetal é determinada pela identificação de três pontos de referência: cabeça, coluna vertebral e fígado. Para a identificação do situs atrial é imprescindível determinar-se a posição da veia cava inferior(VCI) e aorta em relação à coluna vertebral ${ }^{3}$.

Centro de Cardiologia Não Invasiva - São Paulo

Correspondência: Solange Bernardes Tatani - Centro de Cardiologia Não Invasiva Rua Cubatão, 726 - 04013-002 - São Paulo, SP
No situs solitus a aorta estará localizada à esquerda e a VCI à direita em relação aos corpos vertebrais. No situs inversus ocorre o oposto, ou seja, a VCI estará à esquerda da coluna vertebral. No isomerismo direito, a aorta e VCI estão do mesmo lado da coluna, com a veia cava geralmente anterior à aorta. No isomerismo esquerdo, a aorta está usualmente em cima da coluna, estando ausente a VCI.

Para se determinar a anatomia e conexões cardíacas são necessários, de maneira geral, quatro cortes do coração: quatro câmaras, longitudinal do ventrículo esquerdo (VE), arco do canal arterial ou ductal e arco aórtico.

O corte mais facilmente obtido é o quatro câmaras (fig. 1). Nesta projeção, o coração ocupa cerca de um terço do tórax fetal. A aorta descendente (Ao) encontra-se entre a coluna vertebral e o átrio esquerdo (AE). As cavidades atriais têm dimensões semelhantes e pode-se visibilizar a membrana do forame oval, movimentando-se em direção ao AE e a valva de Eustáquio no interior do átrio direito (AD). Pode-se também avaliar a morfologia e dinâmica das valvas atrioventriculares, assim como a conexão atrioventricular. Os dois ventrículos apresentam dimensões semelhantes, mas a geometria contrátil mostra uma dominância do ventrículo direito (VD) ${ }^{4}$. A diferenciação entre os ventrículos pode ser feita pela trabeculação (mais grosseira no VD), pela presença da banda moderadora no VD, pela inserção mais distal da valva tricúspide e pelo aspecto bem definido dos músculos papilares no VE. As espessuras das paredes são semelhantes.

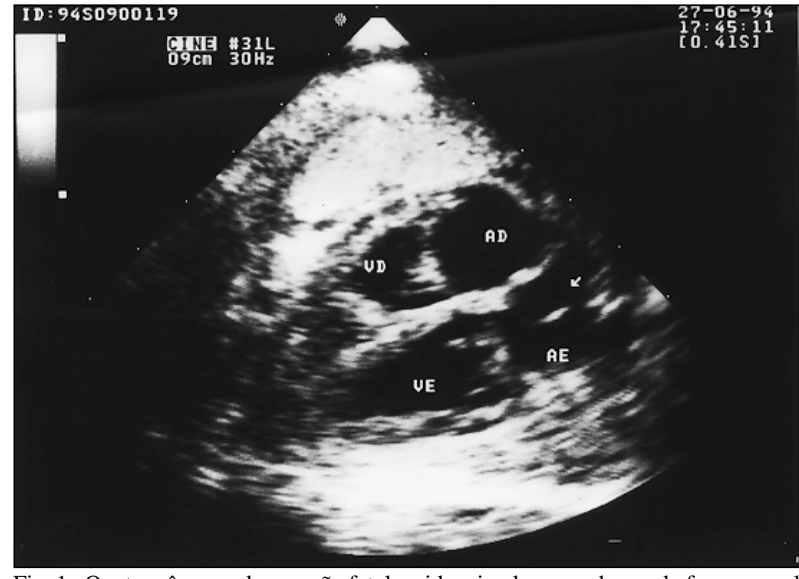

Fig. 1 - Quatro câmaras do coração fetal, evidenciando a membrana do forame oval (seta). AD-átrio direito; AE-átrio esquerdo; VD- ventrículo direito; VE-ventrículo esquerdo. 


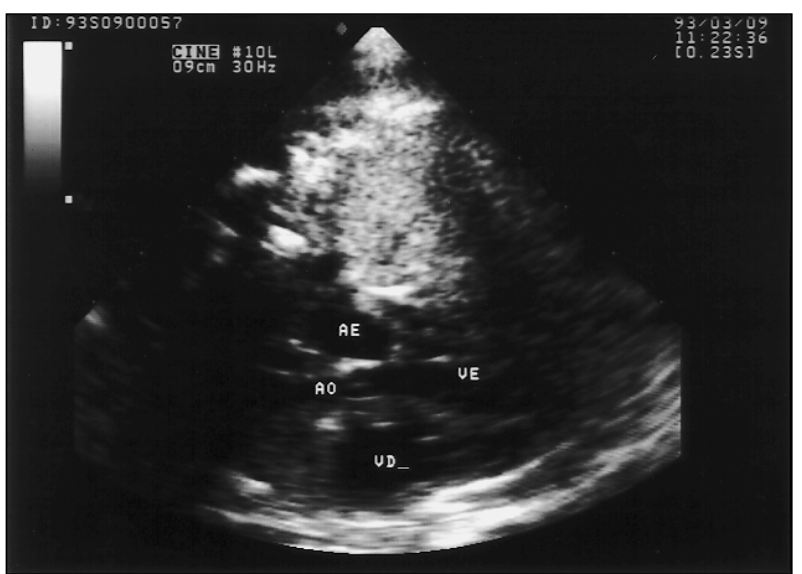

Fig. 2 - Corte longitudinal do ventrículo esquerdo. AE- átrio esquerdo; Ao- aorta; VD- ventrículo direito; VE- ventrículo esquerdo.

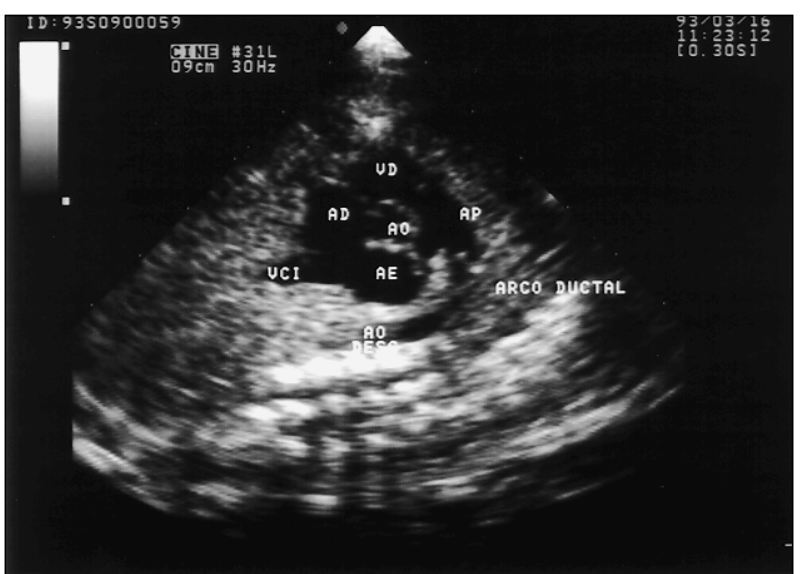

Fig. 3 - Arco do canal arterial ou ductal. AD- átrio direito; $\mathrm{AE}$ - átrio esquerdo; $\mathrm{AP}$ artéria pulmonar; Ao- aorta; DESC- descendente; VCI- veia cava inferior; VDventrículo direito.

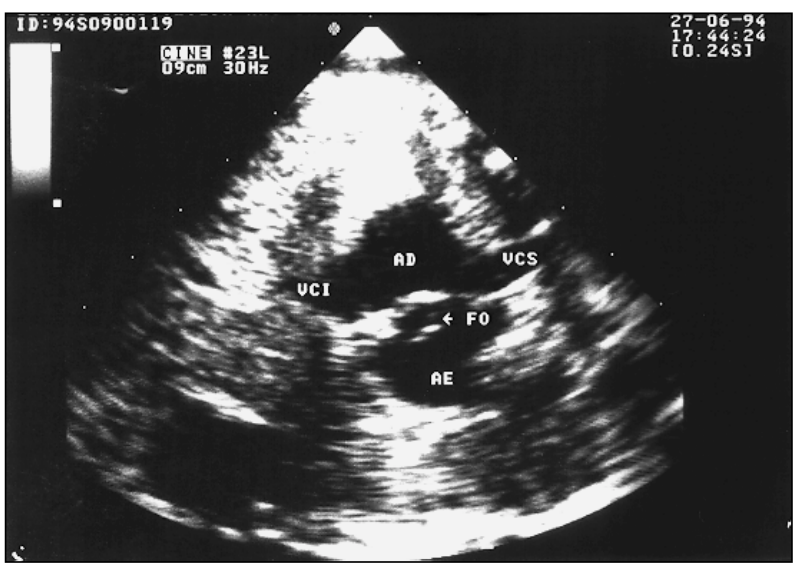

Fig. 4 - Demonstração da drenagem venosa sistêmica. $\mathrm{AD}$ - átrio direito; $\mathrm{AE}$ - átrio esquerdo; FO-forame oval; VCI- veia cava inferior; VCS- veia cava superior.

No corte longitudinal (fig. 2) pode-se identificar as conexões esquerdas do coração fetal. O AE comunica-se através da valva mitral ao VE, de onde emerge a aorta ascendente com a valva aórtica. Pode-se verificar as continuidades mitro-aórtica e aorto-septal.

Na visão do arco do canal arterial (fig. 3) identificam-se as conexões direitas. A VCI desemboca no AD, que se

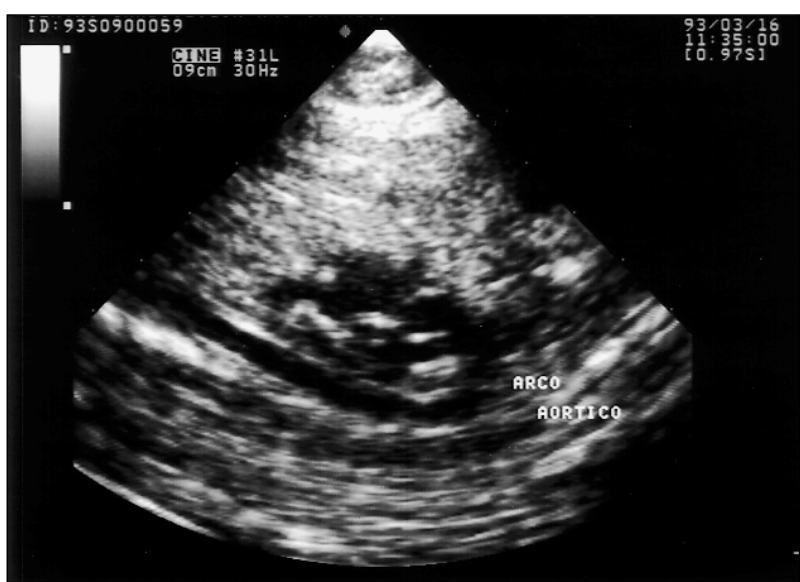

Fig. 5 - Arco aórtico, identificado pelos ramos supra-aórticos.

conecta ao VD através da valva tricúspide. O tronco pulmonar origina-se no infundíbulo direito, podendo-se identificar sua bifurcação e a comunicação da artéria pulmonar esquerda com a Ao, através do canal arterial, formando o arco ductal. A valva pulmonar está situada anteriormente à valva aórtica, sendo possível a avaliação da morfologia e dinâmica de suas válvulas. Nesta projeção é possível também a visibilização da entrada das veias cavas no AD (fig. 4).

Com a colocação do feixe ultra-sônico no plano coronal do feto, obtém-se a imagem do arco aórtico (fig. 5), que normalmente forma um gancho no centro do tórax. É necessário demonstrarem-se os ramos aórticos, originando-se do arco, para diferenciar o arco aórtico do ductal.

A qualidade do exame pode ser limitada pela motilidade fetal (tanto a hipo quanto a hipermobilidade) e pela posição do concepto. A posição occipito-anterior e a localização anterior da placenta são obstáculos por ficarem interpostas entre o transdutor e o coração fetal, dificultando assim, a passagem do feixe ultra-sônico. Outros fatores podem interferir na qualidade das imagens, como a obesidade materna, edema fetal importante e polidrâmnio.

A realização da ECO modo-M de maneira padronizada e reprodutível sucede o reconhecimento e a definição da anatomia cardíaca através da ECO bidimensional. O modoMé mais utilizado para a mensuração das cavidades cardíacas pois permite melhor definição da superfície endocárdica, possibilitando assim uma documentação do crescimento destas cavidades durante a gestação.

Vários estudos ecocardiográficos foram feitos visando estabelecer as dimensões das cavidades cardíacas no feto normal em diferentes idades gestacionais e diâmetros biparietais ${ }^{5-15}$. Os valores normais encontrados variam discretamente, dependendo do método empregado (modo-M ou bidimensional), do corte utilizado (quatro câmaras ou transversal) e da fase do ciclo cardíaco em que foram feitas as medidas (sístole ou diástole). Entretanto, uma verificação comum a esses trabalhos é que as cavidades cardíacas aumentam três a quatro vezes de tamanho da $17^{\mathrm{a}}$ semana ao término da gestação ${ }^{9}$ (tab. I), mostrando um padrão linear de crescimento em relação à idade gestacional (fig. 6) e ao diâmetro biparietal ${ }^{5,7,9,11-15}$ (fig. 7). 


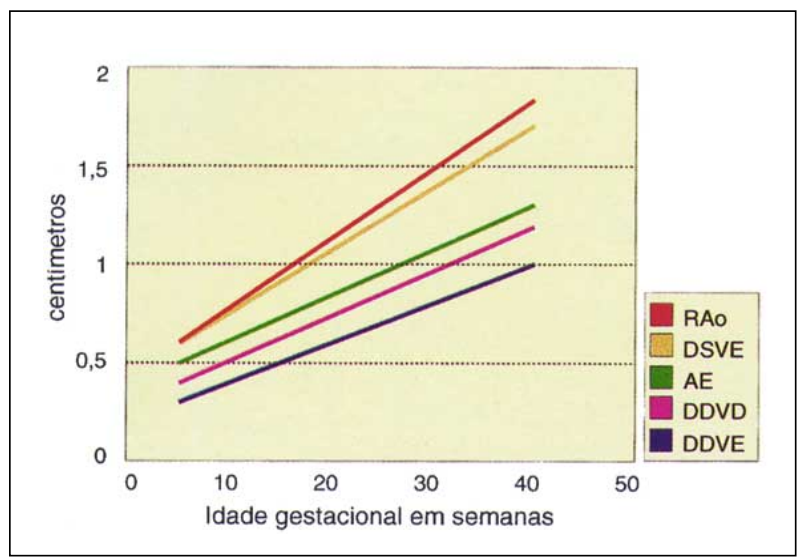

Fig. 6 - Gráfico demonstrando padrão linear de crescimento das câmaras cardíacas fetais em relação à idade gestacional ${ }^{7}$.

Na avaliação dos ventrículos ao modo-M, pode-se observar as suas dimensões e a movimentação de suas paredes. O septo interventricular movimenta-se em direção à parede do VE na sístole, mas pode-se detectar movimentação anômala do septo em até $50 \%$ dos fetos normais. A relação entre as dimensões dos VE e VD é próxima da unidade (variando entre 0,96 e 1,18) e mostra-se constante durante a gestação ${ }^{5-7,9,12,13}$. As dimensões semelhantes dos ventrículos não implicam em volumes ventriculares similares, uma vez que as geometrias ventriculares direita e esquerda são diferentes, assim com a morfologia do trabeculado e das vias de saída ventriculares.

\begin{tabular}{|c|c|c|}
\hline \multicolumn{3}{|c|}{$\begin{array}{c}\text { Tabela I - Variação das dimensões cardíacas }(\mathrm{cm}) \text { da } 17^{\mathrm{a}} \text { à } 40^{\mathrm{a}} \\
\text { semana gestacional }{ }^{9}\end{array}$} \\
\hline & $17^{\mathrm{a}}$ semana & $40^{\mathrm{a}}$ semana \\
\hline Ventrículo esquerdo & 0,4 & 1,6 \\
\hline Ventrículo direito & 0,4 & 1,9 \\
\hline Átrio esquerdo & 0,4 & 1,6 \\
\hline Átrio direito & 0,6 & 1,6 \\
\hline Aorta & 0,24 & 1,0 \\
\hline
\end{tabular}

A partir das dimensões do VE pode-se obter a fração de encurtamento que reflete a função sistólica ventricular fetal e que, em condições normais, mostra-se inalterada com o evoluir da gestação (de $36 \pm 6 \%$ na $20^{\text {a }}$ semana para $32 \pm 5 \%$ ao término) $)^{5,7}$.

As dimensões da aorta e tronco pulmonar também têm sido determinadas. O diâmetro pulmonar excede (em cerca de $12 \%$ ) o da aorta, consistentemente, durante a gestação ${ }^{14,15}$. O maior calibre da pulmonar pode ser consequiência do maior volume sangüíneo ejetado pelo $\mathrm{VD}^{6,15}$.

Os padrões de fluxo no coração fetal podem ser determinados pelo Doppler pulsátil em conjunto com a imagem bidimensional e o mapeamento de fluxo a cores. A velocidade, direção e volume do fluxo sangüíneo podem ser estabelecidos e vão ajudar a determinar a presença de regurgitação, estenose e shunts.

As velocidades dos fluxos mitral e tricúspide não variam significantemente com o evoluir da gestação. A velocidade máxima tricuspídea é discretamente maior que a mitral
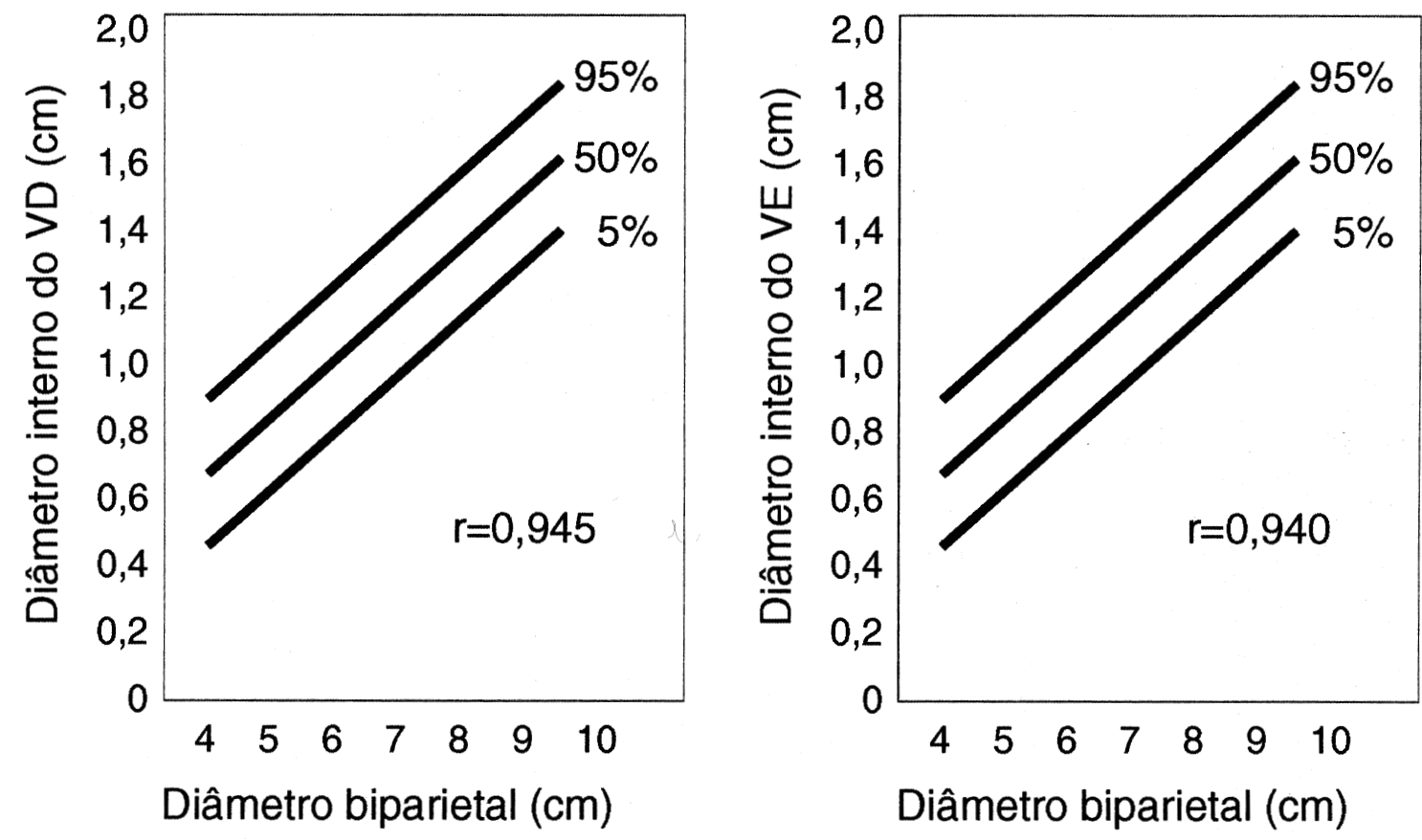

Fig. 7 - Gráficos demonstrando padrão linear de crescimento das câmaras ventriculares fetais em relação ao diâmetro biparietal ${ }^{5}$. 


\begin{tabular}{|lcc|}
\hline \multicolumn{3}{|c|}{ Tabela II - Variação da velocidade máxima dos fluxos pulmonar e } \\
aórtico da $\mathbf{2 0}^{\mathrm{a}} \mathbf{a} \mathbf{4 0} \mathbf{~}^{\mathrm{a}}$ semana gestacional
\end{tabular}

$(0,51 \mathrm{~m} / \mathrm{s}$ vs $0,47 \mathrm{~m} / \mathrm{s})$, não havendo diferença quanto à velocidade média $(11,8 \mathrm{~m} / \mathrm{s} \text { vs } 11,2 \mathrm{~m} / \mathrm{s})^{16}$.

O espectro do fluxo atrioventricular mostra ondas Ee A bem definidas, sendo a onda E a velocidade-pico durante o enchimento ventricular rápido e a onda $\mathrm{A}$, a velocidadepico durante a sístole atrial. A velocidade do fluxo atrioventricular durante a sístole atrial é maior do que a observada durante o enchimento ventricular rápido ${ }^{17}$, ao contrário do que ocorre na fase pós-natal. Tem se postulado que esta dominância da onda A no feto, reflete um comprometimento do relaxamento ventricular, sugerindo maior importância do componente da sístole atrial no enchimento ventricular. A relação entre as ondas E/A para a tricúspide, aumenta de 0,52 na $20^{\mathrm{a}}$ semana para 0,84 ao término da gestação, enquanto que para a mitral a variaçãoé de 0,63 para $0,83^{18}$. Esta relação, que pode ser usada como um índice de função diastólica ventricular, mostra que as propriedades diastólicas dos VEe VD são semelhantes no feto e tendem a mudar durante a gestação.

As velocidades máxima e média do fluxo sangüíneo nas grandes artérias aumentam durante a gestação, sendo a velocidade aórtica discretamente maior que a pulmonar ${ }^{19,20}$ (tab. II). O formato da curva dos fluxos aórtico e pulmonaré similar, porém o tempo de aceleração da pulmonar é mais curto do que o da aorta, não se alterando este padrão com o avançar da idade gestacional ${ }^{21}$.

A velocidade do fluxo no canal arterial é a mais alta do coração fetal, sendo superior à da aorta e artéria pulmonar, variando de 0,6 a $1,2 \mathrm{~m} / \mathrm{s}$ e aumentando durante a gestação. Fluxos diastólicos retrógrados na Ao com velocidade de 0,1 a $0,2 \mathrm{~m} / \mathrm{s}$ são característicos de fluxo ductal.

O cálculo do débito cardíaco do coração fetal é factível, aliando-se o Doppler à ECO bidimensional e modo-M,

\begin{tabular}{|c|c|c|}
\hline \multicolumn{3}{|c|}{$\begin{array}{l}\text { Tabela III - Variação do volume sistólico dos ventrículos da } 20^{\mathrm{a}} \text { à } \\
\qquad 40^{\mathrm{a}} \text { semana gestacional }{ }^{18}\end{array}$} \\
\hline & $20^{\mathrm{a}}$ semana & $40^{\mathrm{a}}$ semana \\
\hline Ventrículo direito & $0,7 \mathrm{~mL}$ & $7,6 \mathrm{~mL}$ \\
\hline Ventrículo esquerdo & $0,7 \mathrm{~mL}$ & $5,2 \mathrm{~mL}$ \\
\hline
\end{tabular}

utilizando-se a velocidade máxima do fluxo aórtico ou pulmonare a área seccional da valva aórtica ou pulmonar, respectivamente. Os estudos realizados mostram que o débito cardíaco de cada ventrículo aumenta exponencialmente durante a gestação (tab. III), com o volume direito excedendo o esquerdo em cerca de $28 \%{ }^{13,18}$.

Em resumo, o uso das técnicas ecocardiográficas no coração fetal nos proporciona a oportunidade de correlacionar os dados anatômicos e hemodinâmicos, possibilitando o estudo da fisiologia cardíaca no período pré-natal. $\mathrm{O}$ VD é o dominante, ejetando a maior proporção de sangue para a Ao, enquanto que o VE supre a cabeça e membros superiores. Provavelmente, decorrente do número reduzido de elementos contráteis, o miocárdio fetal apresenta comprometimento de sua capacidade de relaxamento ${ }^{17}$, resultando em menor habilidade em responder a aumentos da freqüência cardíaca e justificando a insuficiência cardíaca precoce nas taquiarritmias fetais.

Finalmente devemos lembrar que o coração fetal deve ser encarado não estaticamente, mas como uma "estrutura em evolução". A fase de embriogênese cardíaca termina na $7^{\mathrm{a}}-8^{\mathrm{a}}$ semana gestacional, mas a morfogênese desenvolve-se a partir da $15^{\text {a }}$ semana até o término da gestação, com o coração fetal apresentando mudanças progressivas na sua forma, função e tamanho neste período. Como resultado, lesões que estão presentes no início da gestação podem se alterar com o crescimento das cavidades e grandes artérias fetais (por exemplo: um feto com anomalia de Ebstein pode desenvolver atresia pulmonar). Assim, o ecocardiograma fetal deve ser realizado de maneira evolutiva e seriada para possibilitar um diagnóstico preciso e um melhor esclarecimento da fisiopatologia das malformações cardíacas fetais.

\section{Referências}

1. Huhta JC, Rotondo KM - Fetal echocardiography. Semin Roentgen 1991; $26: 5$.

2. Allan LD - A review of fetal echocardiography. Echocardiography $1985 ; 2: 351$ 76.

3. Huhta JC, Smallhorn JF, Macarteney FJ - Two-dimensional echocardiographic diagnosis of situs. Br Heart J 1982; 48: 97.

4. Anzacot A - Analysis of ventricular shape by echocardiography in normal fetuses, newborns and infants. Circulation 1983; 68: 1201.

5. DeVore GR, Siassi B, Platt LD - Fetal echocardiography. IV: M-mode assessment of ventricular size and contractility during the second and third trimesters of pregnancy in the normal fetus. Am J Obstet Gynecol 1984; 150: 981.

6. Sahn DJ, Lange LW, Allen HD et al - Quantitative cross-seccional echocardiography in the developing normal fetus and newborn. Circulation 1980; 62: 588 .

7. St John Sutton MG, Gewitz MH, Shah B et al - Quantitative assessment of growth and function of the cardiac chambers in the normal human fetus: a prospective longitudinal echocardiographic study. Circulation 1984; 69: 645.

8. Wladimiroff JW, Vosters RPL, Vletter W - Ultrasonic measurement of fetal and neonatal ventricular dimensions. Contrib Gynec Obstet 1979; 6: 109.

9. Wladimiroff JW, McGhie J - Ultrasonic assessment of cardiovascular geometry and function in the human fetus. Br J Obstet Gynaecol 1981; 88: 870.

10. Shime J, Gresser CD, Rakowski H - Quantitative two-dimensional 
echocardiographic assessment of fetal cardiac growth. Am J Obstet Gynecol 1986; 154: 294 .

11. Allan LD, Joseph MC, BoydEGCA et al-M-mode echocardiography in the developing human fetus. Br Heart J 1982; 47: 573.

12. Wladimiroff JW, Stewart PA, Vosters RPL-Fetal cardiac structure and function as studied by ultrasound. Clin Cardiol 1984; 7: 239.

13. Tan J, Silverman NH, Hoffman JI et al - Cardiac dimensions determined by crosssectional echocardiography in the normal human fetus from 18 weeks to term. Am J Cardiol 1992; 70: 1459.

14. Reed KL, Anderson CF, Shenker L - Fetal pulmonary artery and aorta: two-dimensional Doppler echocardiography. Obstet Gynecol 1987; 69: 175.

15. Cartier MS, Davidoff A, Warneke LA et al - The normal diameter of the fetal aorta and pulmonary artery. Am J Roentgenol 1987; 149: 1003.

16. Reed KL, Meijboom EJ, Sahn DJ et al - Cardiac Doppler flow velocities in human fetuses. Circulation 1986; 73: 41.
17. Reed KL, Sahn DJ, Scagnelli S et al - Doppler echocardiographic studies of diastolic function in the human fetal heart: changes during gestation. J Am Coll Cardiol 1986; 8: 391 .

18. Kenny J, Plappert T, Doubilet P et al - Effects of heart rate on ventricular size, stroke volume, and output in the normal human fetus: a prospective Doppler echocardiorgaphic study. Circulation 1987; 76: 52.

19. Kenny J, Plappert T, Doubilet Pet al - Changes in intracardiac blood flow velocities and right and left ventricular stroke volumes with gestational age in the normal human fetus: a prospective Doppler echocardiographic study. Circulation 1986; 74: 1208

20. Allan LD, Chita SK, Al-Ghazali W et al- Doppler echocardiographic evaluation of the normal human fetal heart. Br Heart J 1987; 57: 528 .

21. Machado MVL, Chita SK, Allan LD - Acceleration time in the aorta and pulmonary measured by Doppler echocardiography in the mid trimester normal human fetus. Br Heart J 1987; 58: 15. 